\title{
Artemis: guarding small children and, now, the genome
}

\author{
Vicky L. Brandt and David B. Roth \\ Program in Molecular Pathogenesis, Skirball Institute of Biomolecular Medicine, \\ New York University School of Medicine, New York, New York, USA \\ J. Clin. Invest. 111:315-316 (2003). doi:10.1172/JCI200317743.
}

Through careful analysis of young patients exhibiting radiosensitivity and inherited SCID of B and T lymphocytes, Despina Moshous and colleagues discovered a new DNA repair gene two years ago and named it after the Greek goddess Artemis, a guardian of young children and small animals (1). The same group has recently analyzed several patients with hypomorphic Artemis mutations, and their results, published in this issue of the $J C I(2)$, along with a recent report by the group of Fred Alt (3), have proven Artemis to be a guardian of the genome as well. To appreciate the significance of this finding, it is necessary first to review what is known about how our large repertoire of antigen receptors is generated.

\section{The molecular basis of antigen- receptor diversity}

During lymphocyte development, a process called $\mathrm{V}(\mathrm{D}) \mathrm{J}$ recombination cuts, rearranges, and pastes together again separate $\mathrm{V}$ (variable), $\mathrm{D}$ (diversity), and J (joining) gene segments to form a new gene that encodes a unique antigen-binding pocket. The basic contours of this process are depicted in Figure 1a; note that to create a double-strand break in DNA, the RAG proteins form a covalently sealed hair-

\footnotetext{
Address correspondence to: David B. Roth, Program in Molecular Pathogenesis, Skirball Institute of Biomolecular Medicine, New York University School of Medicine, 540 First Avenue, New York, New York 10016, USA. Phone: (212) 263-0945; Fax: (212) 263-5711.

E-mail: roth@saturn.med.nyu.edu.

Conflict of interest: The authors have

declared that no conflict of interest exists.

Nonstandard abbreviations used:

nonhomologous end joining (NHEJ).
}

pin on the coding segment. This hairpin must itself be opened to allow the coding joint to be formed. Mutations that block recombination - say, by preventing hairpin opening and thus coding joint formation (Figure $1 \mathrm{~b}$ ) cause a SCID of both B and T lymphocytes. Mutations that allow joining but cause it to be misregulated (e.g., allowing broken DNA ends to join the wrong partner chromosome) can lead to chromosomal instability and lymphoid malignancies (4).

Proper joining requires the participation of the RAG proteins as well as at least six nonhomologous end joining (NHEJ) factors such as the catalytic subunit of the DNA-dependent protein kinase, DNA-PKcs $(5,6)$. Because NHEJ factors repair all sorts of doublestrand breaks, and loss of their function causes genomic instability through impaired end-joining, these proteins have been called "genome guardians" (4). The precise functions of most NHEJ factors are unknown, but we do know that DNA-PKes is involved in hairpin opening; DNAPKcs mutations affect coding joints more than signal joints (6), and mice lacking DNA-PKcs accumulate hairpins (7). Hairpin opening is a complex process, however, that involves several factors - including Artemis (8).

\section{Small animals, young children, and hairpins}

The connection between NHEJ mutations and genomic stability was established largely in rodents $(9,4)$. Human deficiencies in these genes have not been reported, except for a hypomorphic mutation in ligase IV (10) associated with leukemia and radiosensitivity, but not with a $\mathrm{V}(\mathrm{D}) \mathrm{J}$ recombination defect. (It is likely that severe mutations in end joining factors are embryonic-lethal in humans (11).) Artemis, however, was discovered in children with inherited radiosensitive SCID whose phenotype was strongly reminiscent of DNA-PKcs-deficient mice (severe defects in coding formation but normal signal joints) (1). The specific lesion in coding joint formation immediately suggested that Artemis might be involved in hairpin opening, and indeed, in vitro studies showed that Artemis, when complexed with DNA-PKcs, opens hairpins (12) (Figure $1 \mathrm{~b}$ ). The ultimate proof came with Artemis knockout mice, which, like Artemis-deficient patients, exhibit a defect in coding joint formation with accumulation of hairpins (3). Unlike Artemis-deficient children, however, some of these mice are able to generate lymphocytes containing virtually normal coding joints. This suggests that Artemis does not act alone in opening hairpins, and indeed there has been evidence that the RAG proteins contribute to this process $(8,13)$.

\section{A new genome guardian}

Fibroblasts derived from Artemis-deficient mice show striking genomic instability (3), as expected for cells lacking an NHEJ factor. More importantly, Artemis-deficient patients display aberrant chromosome rearrangements in peripheral blood lymphocytes and develop B cell lymphomas (2). This is perhaps the most exciting aspect of the new work by Moshous et al. - it solidifies the notion that NHEJ mutations are lymphomagenic in humans.

It is clear from the patients and mice that Artemis is critical for the hairpin opening step of $V(D) J$ recombination. But the radiosensitive phenotype suggests that Artemis must also be important for general DNA damage repair. What precise roles might Artemis play? Biochemical studies have established that in addition to hairpin opening, purified Artemis possesses single-strand-specific exonuclease activity and, in the presence of DNAPKcs, endonucleolytic activity on single-strand overhangs as well as hairpins (12). One might therefore expect 


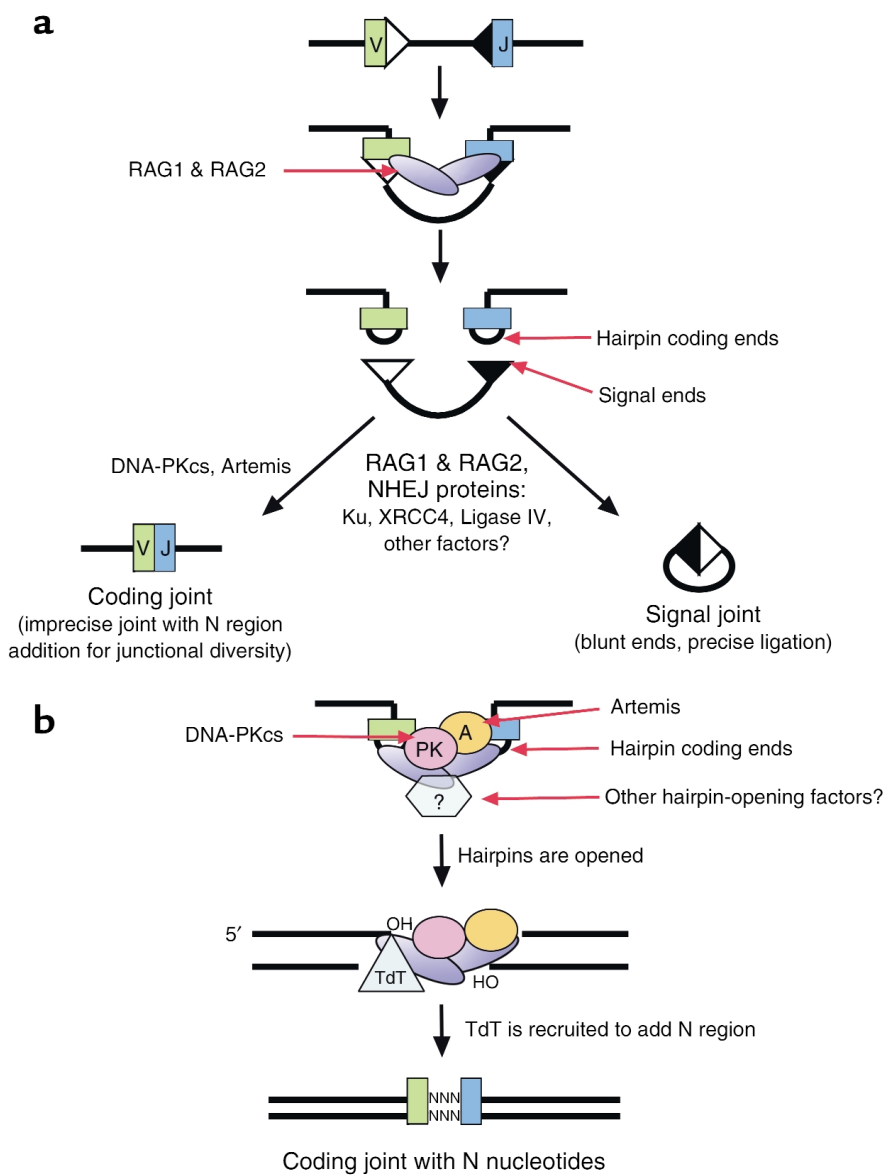

Figure 1

(a) $V(D) J$ recombination is initiated when the RAG1 and RAG2 proteins bind to specific recognition sequences (triangles) that flank the Ig or T cell receptor gene segments (rectangles, here denoted $\mathrm{V}$ and J). The RAG proteins act in concert to cleave the DNA precisely between the specific recognition sequences and the coding segments, producing blunt signal ends and covalently sealed (hairpinned) coding ends. The latter must be opened by endonucleolytic cleavage before forming the coding joint (the rearranged antigen-receptor gene). RAG1/RAG2 and NHEJ factors (listed in the center of the figure) are required for forming both coding and signal joints; DNA-PKcs and Artemis have special roles in creating coding joints, although DNA-PKcs-deficient cells show some abnormal signal joints and a mild decrease in signal joint formation as well. (b) Hairpin opening requires several factors, including Artemis, DNA-PKcs, and possibly the RAG proteins. The hairpins are nicked near the terminus, generating short single-strand extensions; terminal deoxynucleotidyl transferase (TdT) can be recruited to the site to insert $\mathrm{N}$ nucleotides, creating added junctional diversity.

Artemis to participate in nucleolytic processing of damaged DNA ends.

Artemis may also perform nonnucleolytic functions. The rare $\mathrm{T}$ cell receptor rearrangements isolated from patients with hypomorphic Artemis mutations show severely curtailed $\mathrm{N}$ region diversity (2). $\mathrm{N}$ regions are nontemplated nucleotides added to the $3^{\prime}$ ends of RAG-mediated double-strand breaks by the enzyme terminal deoxynucleotidyl transferase (TdT) (Figure $1 \mathrm{~b}$ ) to provide added junctional diversity. $\mathrm{N}$ region addition requires another NHEJ protein, Ku80 (14), probably to recruit other end-processing factors, and Artemis may assist in this recruiting. Nonetheless, Artemisdeficient mice showed no decrease in $\mathrm{N}$ regions (3). These differences between patients and mice - i.e., mice produce some coding joints and add $\mathrm{N}$ regions while patients do not - could reflect species-specific differences in nonhomologous end-joining that should be further explored.

The clinical import of these studies is that we now have several candidate genes to examine in patients with lymphoid neoplasms: the known NHEJ factors and perhaps the RAG proteins, which are also necessary for the proper joining of $V(D) J$ recombination intermediates. Although some of these proteins have been associated with cancer in mice, evidence of their connection to lymphoma in humans has been less forthcoming even though lymphoid neoplasms are among the most common human cancers. We hypothesize that hypomorphic mutations in any of these genes could deregulate joining sufficiently to increase the incidence of oncogenic rearrangements. Inherited or acquired mutations in NHEJ factors could be important risk factors for developing lymphoid neoplasms and perhaps other types of cancer as well.

1. Moshous, D., et al. 2001. Artemis, a novel DNA double-strand break repair/V(D)J recombination protein, is mutated in human severe combined immune deficiency. Cell. 105:177-186.

2. Moshous, D., et al. 2003. Partial T and B lymphocyte immunodeficiency and predisposition to lymphoma in patients with hypomorphic mutations in Artemis. J. Clin. Invest. 111:381-387. doi:10.1172/JCI200316774.

3. Rooney, S., et al. 2002. Leaky scid phenotype associated with defective $\mathrm{V}(\mathrm{D}) \mathrm{J}$ coding end processing in Artemis-deficient mice. Mol. Cell. 10:1379-1390.

4. Roth, D.B., and Gellert, M. 2000. New guardians of the genome. Nature. 404:823-825.

5. Brandt, V.L., and Roth, D.B. 2002. A recombinase diversified: new functions of the RAG proteins. Curr. Opin. Immunol. 14:224-229.

6. Grawunder, U., and Harfst, E. 2001. How to make ends meet in V(D)J recombination. Curr. Opin. Immunol. 13:186-194.

7. Roth, D.B., Menetski, J.P., Nakajima, P.B., Bosma, M.J., and Gellert, M. 1992. V(D)J recombination: Broken DNA molecules with covalently sealed (hairpin) coding ends in scid mouse thymocytes. Cell. 70:983-991.

8. Schlissel, M.S. 2002. Does artemis end the hunt for the hairpin-opening activity in $\mathrm{V}(\mathrm{D}) \mathrm{J}$ recombination? Cell. 109:1-4

9. Zhu, C., et al. 2002. Unrepaired DNA breaks in p53-deficient cells lead to oncogenic gene amplification subsequent to translocations. Cell. 109:811-821.

10. Riballo, E., et al. 1999. Identification of a defect in DNA ligase IV in a radiosensitive leukaemia patient. Curr. Biol. 9:699-702.

11. Li, G., Nelsen, C., and Hendrickson, E.A. 2002 Ku86 is essential in human somatic cells. Proc. Natl. Acad. Sci. USA. 99:832-837.

12. Ma, Y., Pannicke, U., Schwarz, K., and Lieber, M.R. 2002. Hairpin opening and overhang processing by an Artemis/DNA-dependent protein kinase complex in nonhomologous end joining and V(D)J recombination. Cell. 108:781-794.

13. Huye, L.E., Purugganan, M.M., Jiang, M.M., and Roth, D.B. 2002. Mutational analysis of all conserved basic amino acids in RAG-1 reveals catalytic, step arrest, and joining-deficient mutants in the V(D)J recombinase. Mol. Cell. Biol. 22:3460-3473.

14. Bogue, M.A., Wang, C., Zhu, C., and Roth, D.B. 1997. V(D)J recombination in Ku86-deficient mice: Distinct effects on coding, signal, and hybrid joint formation. Immunity. 7:37-47. 